\title{
AnÁlise das ESTRATÉgias UTILIZAdAS EM UM GRUPO TERAPÊUTICO PEDAGÓGICO PARA AUXILIAR O DESENVOLVIMENTO DA LINGUAGEM ESCRITA EM CRIANÇAS COM DEFICIÊNCIA AUDITIVA
}

\author{
A NALYSES OF STRATEGIES U SED IN A PEDAGOGIC THERAPEUTIC GROUP TO \\ ENHAN CE THE DEVELOPMENT OF WRITING IN HEARING IM PAIRED CHILDREN
}

Janaína Luciane DUARTE ${ }^{1}$

Joseli Soares BRAZOROTTO²

\begin{abstract}
RESUM O: o presente estudo teve como objetivos avaliar e acompanhar o desenvolvimento da linguagem escrita e analisar as estratégias utilizadas durante 01 ano de intervenção pedagógica em grupo para o desenvolvimento da linguagem escrita de quatro crianças com deficiência auditiva, usuárias de A parelho de Amplificação Sonora Individual (AASI) e/ ou Implante Coclear (IC) com idades entre 05 e 07 anos, atendidas no Centro Educacional do Deficiente Auditivo do Hospital de Reabilitação de Anomalias Craniofaciais da Universidade de São Paulo, em Bauru-SP. Para a consecução dos objetivos foram realizados os seguintes procedimentos: submissão do projeto de pesquisa ao Comitê de Ética em Pesquisa da instituição, revisão da literatura, análise das estratégias para a intervenção, categorização das habilidades de audição e de linguagem oral de cada criança após avaliações da percepção auditiva da fala e de linguagem oral realizadas por um fonoaudiólogo, bem como avaliação da linguagem escrita de acordo com protocolos específicos, no pré e pós-intervenção. As estratégias foram aplicadas na situação de grupo, mediadas pela pedagoga-pesquisadora e registradas com câmera fixa, instal ada em tripé. Realizada a análise das estratégias por meio de transcrição e categorização das mesmas bem como a análise quantitativa e qualitativa das avaliações de cada criança, buscando situar seu nível de desenvolvimento de escrita de acordo com a literatura. Observou-se que as crianças avançaram nas fases de desenvolvimento da escrita e concluiu-se que as estratégias terapêuticas aplicadas despertaram maior envolvimento, confiança e habilidade das crianças em relação à linguagem escrita, mostrando assim, sua utilidade na intervenção pedagógica em grupo.
\end{abstract}

PALAVRAS-CHAVES: educação especial; deficiências da audição; aquisição da escrita; educação especial.

ABSTRACT: the aims of the present study were to evaluate and to accompany the development of writing in an educational and therapeutic group setting with four children between the ages of 5 and 7 years with mild to profound hearing loss, all of whom use hearing aids or have cochlear implants. The objective was to analyze strategies used during such intervention for the development of writing language in this group, during 1 year. The research project was approved by the Ethics Committee of the Hospital of Abnormalities and Craniofacial Rehabilitation of the University of São Paulo and the study was conducted with the parents' consent. The following procedures were undertaken: literature review and analysis of strategies for the intervention, assessment of hearing and language abilities of each child, as well as evaluation of the writing language following specific protocols, before and after intervention. The strategies were applied in group situation, mediated by the educator-researcher and recorded with camera fixed on a tripod. The strategies were transcribed and analyzed. Quantitative and qualitative analysis of each child's evaluations was done, so as to understand the child's level of writing development in accordance with the literature. The

\footnotetext{
${ }^{1}$ Pedagoga, especialista em psicopedagogia, habilitada em educação especial: área de deficiência auditiva. Centro Educacional do Deficiente Auditivo do Hospital de Reabilitação de Anomalias Craniofaciais da Universidade de São Paulo - janainaluciane@yahoo.com.br

2 Professora Adjunta de Fonoaudiologia da Universidade Federal do Rio Grande do Norte - UFRN (Centro de Ciência da Saúde - CCS - Departamento de Cirurgia) - joselisb@usp.br
} 
results showed that all children showed progress in their writing development. The conclusion was that the therapeutic strategies that were applied enabled greater involvement, confidence and abilities regarding written language, pointing to the usefulness of group educational intervention.

KEYWORDS: Special Education; Hearing Impairment; Writing Acquisition; Special Education.

\section{INTRODUÇÃo}

Sabe-se que a leitura e a escrita desempenham papéis fundamentais em nossas vidas, considerando que os signos linguísticos (oral e escrito) são o meio básico para dominar e dirigir a constituição das funções psíqui cas superiores.

Além disso, deparamo-nos com um mundo no qual a maioria das informações é acessada através da leitura e transmitida por meio da escrita.

Qualquer intercorrência no desenvolvimento linguístico nos primeiros anos de vida pode interferir consideravelmente no processo de desenvolvimento da linguagem escrita, o que acarreta em um afastamento do conteúdo que pode ser acessado pela criança por meio da escrita, fato que pode limitar o crescimento global de um sujeito crítico e socialmente ativo.

O aprendizado da linguagem escrita pode ocorrer espontaneamente, através da experiência pessoal da criança ou de maneira sistemática, através da aprendizagem em sala de aula e ainda por meio de apoio pedagógico especializado.

Desta forma, o papel do outro, o ambiente familiar e o aspecto lúdico são essenciais para o aprendizado efetivo da linguagem escrita.

A aquisição e desenvolvimento de habilidades específicas tornam-se importantes para o aprendizado inicial da leitura e escrita, tais como: a percepção visual e auditiva, a compreensão dos conceitos representados pelas palavras e frases, as memórias de trabal ho emediata eas habilidades metalinguísticas (como a consciência fonológica e consciência sintática).

$\mathrm{Na}$ criança com deficiência auditiva podem ser encontradas dificuldades durante o processo de aquisição da linguagem oral e escrita, dado seu déficit sensorial, o que a torna uma criança de risco para a aquisição e desenvolvimento da linguagem escrita e, por conseguinte, do conteúdo da aprendizagem escolar em geral.

A poiando-se na oralidade e/ ou gestos para escrever, a criança com deficiência auditiva necessita de constante reforço analítico e de grande variedade de recursos para impulsionar a construção de sua linguagem escrita, em um momento em que ainda passa pela construção eaprimoramento de sua linguagem oral. 
Para uma intervenção efetiva relacionada à linguagem escrita, a opção educacional escol hida pela família para o desenvolvimento linguístico da criança com deficiência auditiva deverá ser considerada e os programas de reabilitação para o desenvolvimento da audição e linguagem oral (A bordagem Aurioral) poderão contar com atuação terapêutica para promover a aquisição e desenvolvimento da linguagem escrita.

Neste contexto, o trabalho pedagógico dentro da equipe interdisciplinar de reabilitação é um importante facilitador para o acesso otimizado à linguagem escrita pela criança.

Justificam-se, pois, estudos que realizem a análise das estratégias terapêuticas utilizadas como meio de auxiliar a aquisição da linguagem escrita em crianças com deficiência auditiva, participantes de um programa de reabilitação Aurioral.

\section{ReVISÃo da Literatura}

Quando uma criança ouvinte inicia seu interesse e aprendizado da linguagem escrita, geralmente já possui uma linguagem ampla dominando seu conjunto de regras e usos (BARRERA, 2000), o que geralmente não acontece com seu colega com deficiência auditiva, que pode apresentar desenvolvimento de linguagem oral imaturo para sua faixa etária.

Diversas pesquisas buscam relacionar as habilidades envolvidas mais intimamente no processo de apropriação da linguagem escrita em diversas populações, destacando-se os estudos de Capovilla e Capovilla, (2000), Barrera,(2000), Brazorotto (2002), entre outros.

Zorzi (1998) observa que dada a complexidade deste sistema de representação (a escrita), é possível observar-se um processo gradual de apropriação pela criança, sendo que alguns aspectos importantes devem ser compreendidos na aprendizagem da escrita:

- a relação entre letras e sons (um som pode ser representado por uma letra, uma mesma letra pode representar vários sons e um mesmo som pode ser escrito por várias letras);

- a correspondência quantitativa entre letras e sons: cada palavra se escreve com um certo número de letras, que nem sempre corresponde ao número de fonemas que a compõem;

- as variações entre o modo de pronunciar as palavras e a maneira de escrevê las;

- a posição de cada letra no espaço gráfico e direção da escrita;

- a linearidade, que corresponde ao fato de uma letra ser escrita após a outra;

- a segmentação, que indica as pausas e segmentações da escrita. 
Deste modo, a apropriação da língua escrita pela criança demonstrase um processo gradativo, em que várias estratégias são utilizadas para a compreensão e utilização do código escrito. Permeando a aprendizagem da linguagem escrita estão, portanto, as habilidades de metalinguagem, que permitem à criança compreender a natureza e estruturas deste código tão complexo (ZORZI, 2000).

As pesquisas de Ferreiro (1985) e Ferreiro e Teberosky (1986) consideraram que as todas as crianças passam por diferentes níveis de conceptualização da escrita e que o ritmo de progressão nesse processo depende basicamente da quantidade e qualidade de contato com a linguagem escrita a que a criança tem acesso.

Considerando que a aquisição da fala, da leitura e da escrita são dificuldades geralmente encontradas pelas crianças com deficiência auditiva, devido ao déficit na recepção sensorial auditiva das informações, destaca-se que tais dificuldades podem provocar um baixo desempenho na aquisição de aspectos importantes para a formação de um indivíduo socialmente ativo, crítico e participativo na sociedade o qual está inserido.

Trenche rel atou, sobrea aprendizagem da leitura e escrita em crianças com deficiência auditiva:

\begin{abstract}
Um fator de desaceleração no processo de desenvolvimento da leitura eescrita éa incorporação da auto-imagem de que não são sufici entementecompetentes para dar conta das tarefas rel acionadas a ler e escrever. N essa circunstância, o escrito passa a ser visto como algo inatingível, já que sua produção na visão tradicional de linguagem está à margem das normas que regem o uso da língua. (TREN CHE, 1998, p.10-19).
\end{abstract}

Lopes (1989) comenta quelevando em consi deração as variáveis como o diagnóstico e atendimento precoce, os graus de perda auditiva, a adaptação aos recursos el etrônicos auxiliares à audição (aparel hos de amplificação sonora individual, implantes cocleares e ainda, sistemas de frequência modulada) e uma abordagem voltada para facilitar o desenvolvimento natural da linguagem oral, facilita-se o surgimento dos níveis de conceituação da escrita na criança com deficiência auditiva.

Segundo França et al. (2004), Salgado e Capellini (2004), Leitão, Hogben e Fletcher (1997) crianças com dificuldades de fala e linguagem, como as crianças com deficiência auditiva, são consideradas crianças de risco para a aprendizagem por apresentarem dificuldades nas habilidades de consciência fonológica e com isso dificuldades na linguagem escrita.

Os mesmos autores e outros como Desjardin, Ambrose e Eisenberg (2008), James et al. (2007), Góes e Aragão (1992), Miller (2000), Moores (1997) destacaram que a aquisição da linguagem oral destas crianças requer atenção 
especial para que suas dificuldades não limitem o processo de construção da linguagem escrita.

Outro fator relevante para a aprendizagem da linguagem escrita é a abordagem de (re) habilitação na qual a criança com deficiência auditiva está inserida.

Petrechen (2001) analisou o processo de desenvolvimento da escrita de crianças com deficiência auditiva em reabilitação Aurioral na fase inicial do processo de alfabetização e concluiu que as crianças com deficiência auditiva reabilitadas nesta abordagem passam pelas mesmas etapas de desenvolvimento da escrita pelas quais passam as crianças ouvintes. A autora notou que as crianças avaliadas que foram reabilitadas por meio de terapia fonoaudiológica individual e pedagógica grupal e usaram efetivamente os aparelhos de amplificação sonora individual (AASI) alcançaram o nível alfabético de escrita na mesma idade em que crianças ouvintes.

É importante considerar também que o desenvolvimento das habilidades de leitura e escrita pelas crianças com deficiência auditiva pode leválas a um aprimoramento de sua linguagem oral. Tal relação foi destacada por Bueno (2003).

Pesquisas de intervenção nesta área na população de crianças com deficiência auditiva ainda são limitadas e alguns estudos relacionados à eficácia de programas de treino das habilidades específicas para o desenvolvimento da leitura e escrita Bernardino-Júnior (2006), Byrne (1995), Capellini e Ciasca (2000), Capellini, Padula e Ciasca (2004), Capovilla e Capovilla (2000), Capovilla e Capovilla (2002), Gillon e Dood (1997), Jardini e Souza (2006), Poskiparta, Niemi e Vauras (1999), Schneider et al. (1999), Valério (1998) foram desenvolvidos com populações distintas tais como crianças com dislexia ou com dificuldades de aprendizagem, demonstrando resultados satisfatórios, com ganhos no desenvolvimento da linguagem escrita.

\section{Bjetivos}

Avaliar individualmente e acompanhar o desenvolvimento da linguagem escrita das quatro crianças com deficiência auditiva durante um ano do trabalho terapêutico pedagógico em grupo.

Analisar as estratégias utilizadas durante a intervenção pedagógica voltada para o desenvolvimento da linguagem escrita, em um grupo de quatro crianças com deficiência auditiva, usuárias de AASI (A parelho de Amplificação Sonora) e/ ou Implante Coclear, com idades entre cinco e sete anos. 


\section{M ATERIAL E MÉTOdo}

\subsection{Sujeitos}

Tabela 1 - Sujeitos do estudo.

\begin{tabular}{ccccc}
\hline Crianças & G.R.L. & L.V.B. & S.A.B. & T.C. \\
\hline Idade & 7 anos & 7 anos & 5 anos & 7 anos \\
Gênero & masculino & masculino & feminino & feminino \\
$\begin{array}{c}\text { Tipo da Perda } \\
\text { Auditiva }\end{array}$ & DANS & DANS & DAM & DANS \\
$\begin{array}{c}\text { Grau } \\
\text { Escolaral }\end{array}$ & bilateral & bilateral & bilateral \\
Tipo de Dispositivo & AASI & AASI & AASI & IC/AASI \\
\hline
\end{tabular}

Fonte: Dados extraídos dos prontuários dos sujeitos da pesquisa.

Legenda: DANS- Deficiência Auditiva Neurossensorial; DAM - Deficiência Auditiva Mista; AASIA parel ho de Amplificação Sonora Individual; IC- Implante Coclear; E.F.- Ensino Fundamental; E.I.:Educação Infantil.

\subsection{InStRUmentos}

- Prova das quatro palavras e uma frase (GROSSI, 1988): avaliação da escrita sob ditado de 12 palavras e três frases, com objetivo de analisar o nível de desenvolvimento de escrita, baseados em níveis e interníveis, propostos pela autora.

- Avaliação da escrita sob ditado, adaptada de Pinheiro (1994): avaliação da escrita sob ditado de 18 palavras de alta e baixa freqüência.

- Avaliação da escrita espontânea e análise de acordo com as etapas de desenvolvimento da escrita, baseadas em Ferreiro (1985).

Cabe destacar que avaliações específicas das habilidades auditivas e da linguagem oral foram aplicadas com as crianças participantes para posterior categorização de suas habilidades de audição e de linguagem oral, contudo, tais avaliações não foram descritas, visto que não foi objetivo do presente estudo correlacionar os dados de audição elinguagem ao desenvolvimento da linguagem escrita, embora se considere que estas habilidades possuem inter-relação. 


\subsection{Situação}

As avaliações no pré-intervenção e pós-intervenção foram realizadas individualmente e as sessões terapêuticas foram realizadas em grupo, e contaram com a participação do experimentador, caracterizando o presente estudo como pesquisa-ação.

\subsection{Procedimentos}

1. Leitura e explicação da carta de informação ao sujeito da pesquisa e Termo de Consentimento Livre e Esclarecido aos pais das crianças participantes;

2. Coleta de dados pré-intervenção;

3. Coleta dos dados durante a intervenção pedagógica: quinzenalmente as sessões terapêuticas foram registradas em vídeo com câmera em posição fixa, instalada em tripé ;

4. Coleta dos dados pós-intervenção.

\section{Resultados}

As avaliações de cada criança foram analisadas quantitativamente e qualitativamente, descritas abaixo e expostas nos Gráficos 1 e 2 .

A s estratégias registradas em vídeo foram analisadas qualitativamente no intuito de determinar quais os tipos de estratégias foram mais efetivas ou produtivas como auxiliares no desenvolvimento da linguagem escrita do grupo, discutindo suas implicações para a reabilitação de crianças com deficiência auditiva.

Gráfico 1 - Porcentagens de acertos na escrita sob ditado de palavras (adaptado de Pinheiro, 1994).

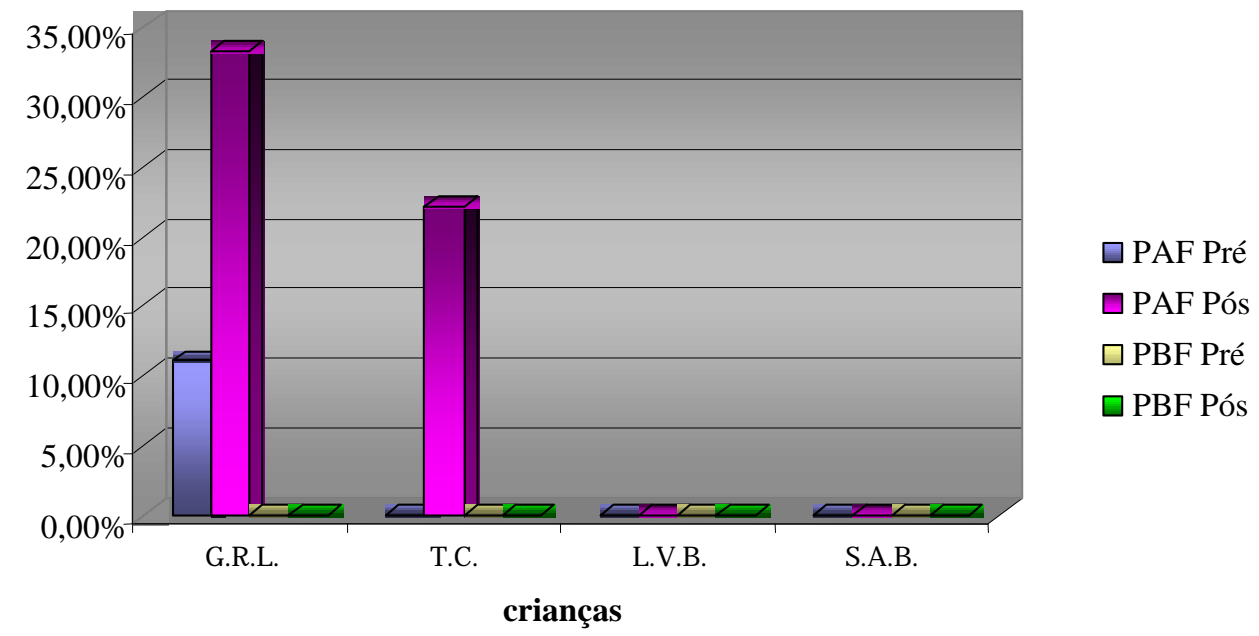


Observa-se, no Gráfico 1, a porcentagem de acertos na escrita sob ditado de pal avras deal ta ebaixa frequência, nas avaliações prée pós-intervenção. Em concordância com o estudo de Pinheiro (1994) a escrita de palavras de alta frequência foi tarefa mais fácil do que a escrita de palavras de baixa frequência para as crianças, o que foi também constatado por Brazorotto (2002), que avaliou crianças com deficiência auditiva reabilitadas no mesmo programa do qual participam os sujeitos do presente estudo.

Para se demonstrar a evolução de cada criança em relação a conceptualização da escrita, convencionou-se a atribuição de pontos entre as fases e interfases, da seguinte maneira:

- 7 pontos- Fase Alfabética

- 5 pontos- Fase Silábico-Alfabética

- 3 pontos- Fase Silábica

- 1 ponto - Fase PréSilábica

Para cada avanço de subfase foram adicionados à pontuação total da criança 0,5 pontos, de forma que uma criança que não evoluiu de fase, mas evoluiu nas interetapas da fase categorizadas por Grossi (1987) teve seu desempenho claramente visualizado.

Gráfico 2 - Evolução nas etapas de conceptualização da escrita das crianças participantes durante um ano.

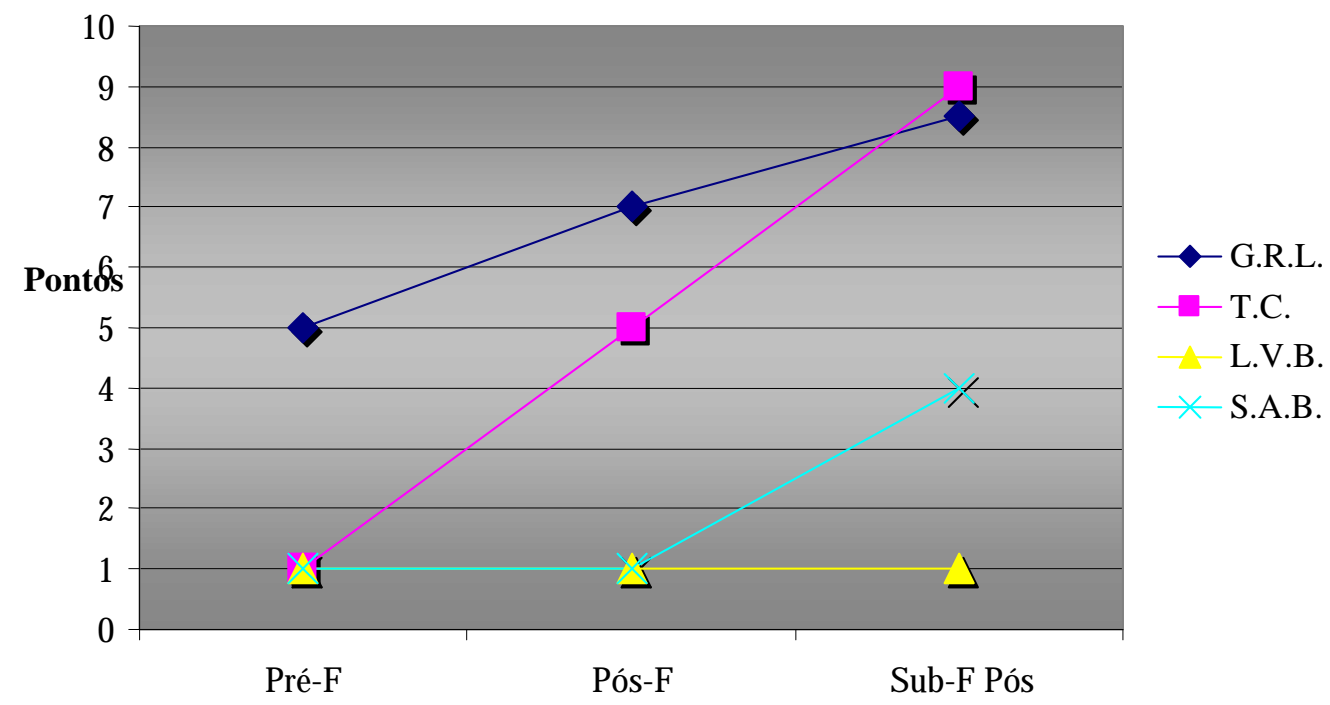

Fases da escrita 
O gráfico 2 apresenta os níveis de desenvolvimento da escrita (FERREIRO, 1985) identificados após análise das avaliações realizadas na pré intervenção e pós-intervenção, bem como o crescimento entre as subfases de cada etapa, de acordo com a análise proposta por Grossi (1987).

\subsection{ESCRIÇão E ANÁLISE dAS ESTRATÉGIAS UTILIZADAS}

De modo a organizar a análise das estratégias utilizadas no trabalho pedagógico em grupo com as crianças, delineou-se uma tipologia para as estratégias utilizadas e anal isadas, facilitando assim, a compreensão dos objetivos de cada uma das estratégias selecionadas.

- Estratégias exploratórias: estratégias que visam explorar os usos e funções da linguagem escrita com as crianças, levando-as a lançarem-se no mundo da leitura e da escrita, compreendendo seu significado social;

- Estratégias baseadas em vivências: estratégias que buscam al iar as experiências e a ampliação da linguagem oral vivenciadas pelas crianças às experiências relacionadas às leituras e especialmente ao registro escrito de tais vivências;

- Estratégias de metalinguagem: estratégias analíticas, nas quais a criança deverá ser envolvida a prestar atenção aos aspectos da linguagem aos quais ela ainda não atentava. Ex: estratégias sobre a consciência fonológica (consciência de sílabas, rimas, fonemas e relação grafema-fonema) e sobre a consciência sintática (organização de frases).

Tais estratégias envolveram o uso de crachás, leitura e contagem de história em grupo, visita à biblioteca municipal, zoológico da cidade, banca de revistas, vivência com as placas de sinalização, construção de história em quadrinhos, leitura coletiva a partir de caça-palavras e construção de texto a partir de receitas, leitura de rótulos e escrita sob ditado utilizando os rótulos, registro de cartão, bilhete e carta, cal endário, brincadeiras com rimas, al iterações e tarefas de consciência fonológica e sintática.

\section{DIscussÃo}

O trabal ho de reabilitação de crianças com deficiência auditiva em uma Abordagem Aurioral (BEVILACQUA; FORMIGONI, 1998; BEVILACQUA; MORET, 2005) enfatiza o uso da audição residual otimizada pelos dispositivos auxiliares à audição, com o objetivo do desenvol vimento da audição para o al cance da competência linguística oral destas crianças e considera-se esta uma forma importante de a criança conquistar sua inclusão acadêmica e social, vivendo como um sujeito ativo em sua comunidade.

Em concordância com a afirmação de Moreira (1995), de que a linguagem escrita é parte fundamental para o desenvolvimento de um sujeito socialmente ativo, torna-se, pois, importante, que os programas de reabilitação 
voltados às crianças com deficiência auditiva invistam também no trabalho de otimização do acesso à linguagem escrita, trazendo mais próximo da criança um ambiente facilitador e ainda preparando a mesma para diferentes contatos e usos da linguagem escrita, o que pode aprimorar também o seu desempenho em linguagem oral (BUENO, 2003).

Estudos específicos neste campo de pesquisa são ainda escassos e poucos são os trabalhos de intervenção desenvolvidos em relação à linguagem escrita nesta população (PETRECHEN, 2001; BALIEIRO; NOVAES, 2005), justificando-se, pois, a necessidade de ampliação e aprofundamento dos mesmos, inclusive das investigações com crianças com deficiência auditiva usuárias de implante coclear (WATSON, 2002; JAMES et al. 2007).

Assim, os estudos que relatam o uso de estratégias para melhorar a relação e o desempenho de crianças em relação à linguagem escrita geralmente são descritos em populações de crianças ouvintes de baixa renda, com distúrbios ou dificuldades de aprendizagem, que assim como as crianças com deficiência auditiva, são crianças de risco para o desenvolvimento da linguagem escrita, como apontam Byrne (1995), Capellini; Padula; Ciasca (2004), Capovilla e Capovilla (2000; 2002), Gillon; Dood (1997), Poskiparta; N iemi; Vauras (1999), Schneider et al. (1999), Valério (1998) e outros.

$\mathrm{Na}$ busca por propostas de atividades e estratégias para auxiliar o desenvolvimento da linguagem escrita em crianças, os trabalhos de Almeida e Duarte (2003), Balieiro e Novaes (2005), Capovilla e Capovilla (2000) e Condemarin et al. (1997) demonstraram ser uma opção viável para a inspiração das estratégias elencadas para o trabal ho em grupo terapêutico pedagógico, de modo quetodas as estratégias vivenciadas no grupo foram estimulantes eeficazes, embora ainda devam ser lapidadas eampliadas, verificando-se sua aplicabilidade em outros grupos terapêuticos, considerando faixa etária, escolaridade, nível de desenvolvimento de audição e de linguagem oral .

A partir das avaliações realizadas no pré e pós-intervenção com as crianças notou-se que houve um progresso quanto ao desenvolvimento de sua linguagem oral e escrita, o que possibilitou hipotetizar que assim como para as crianças ouvintes existe uma ligação entre o desenvolvimento linguístico oral e a construção da escrita para crianças com deficiência auditiva. Tal afirmação corrobora com os pressupostos defendidos por Barrera (2000).

Ainda em relação ao desenvolvimento da linguagem escrita das crianças acompanhadas no presente estudo, notou-se que estas passaram pelas mesmas etapas de conceptual ização da escrita Ferreiro (1985), Ferreiro; Teberosky (1986), Grossi (1987) por que passam as crianças ouvintes, nas tentativas e erros de entender a natureza alfabética de nosso sistema de escrita.

Dada a complexidade deste sistema (ZORZI, 2000) as crianças de risco para o desenvolvimento da linguagem escrita devem ser mergulhadas no 
universo da leitura e escrita, o que certamente as tornará mais confiantes para lançarem-se em atividades que envolvam o material escrito bem como para seus diversos usos, o que foi observado nas quatro crianças participantes do estudo, especialmente durante as estratégias dos tipos vivência eexploratória. As crianças demonstraram ter adquirido mais confiança em sua capacidade de ler e escrever, mesmo para o sujeito L.V.B., que não al cançou mel hora significativa no processo de conceptualização da escrita.

Além disso, o fato de terem recebido instruções específicas sobre o sistema de escrita (relações grafema-fonema, habilidades de metalinguagem) por meio de atividades prazerosas envolvendo também o trabalho com a audição, demonstrou ser facilitador em seu processo de desenvolvimento da linguagem escrita.

Desta forma, as estratégias do tipo metalinguagem tornaram possível que as crianças participantes atentassem para aspectos importantes da linguagem escrita (COWIN ; MANN , 1989) aos quai s elas possivelmentelevariam mais tempo para acessar que seus colegas ouvintes, o que deve ser levado em conta na organização de programas de intervenção para crianças com deficiência auditiva.

N otou-se, finalmente, que a prática das estratégias em grupo gerou progressos não apenas das habilidades de leitura e escrita, mas também das habilidades de audição e linguagem oral de forma motivadora, de modo que a própria prática das atividades gerou a recompensa para as crianças participantes.

\section{Conclusões}

O presente estudo apenas iniciou a busca por conhecimentos mais específicos a respeito das propostas de intervenção pedagógica com crianças com deficiência auditiva, tendo sido este ainda limitado a uma faixa etária alvo (cinco a sete anos), merecendo ser aprofundadas as pesquisas a este respeito.

A s estratégias utilizadas nesta pesquisa são apenas um guia, etambém deverão ser ampliadas e reaplicadas, com mais recursos e mais tempo.

Assim, as principais considerações a partir da presente pesquisa foram:

- O desenvolvimento da linguagem escrita aconteceu para todas as crianças durante o ano de intervenção, porém, para G.R.L. e T.C. foi mais veloz.

- As crianças demonstraram passar pelas mesmas etapas de conceptualização da escrita que as crianças ouvintes.

- O desenvolvimento em relação à linguagem escrita pode ser observado nas avaliações pós-intervenção e mostrou-se nítida, na avaliação qualitativa das estratégias, a melhora na relação de contato das crianças com o material escrito, no entendimento das mesmas sobre o processo de leitura e de escrita, seus usos e funções. 
- As estratégias terapêuticas aplicadas demonstraram ser compatíveis aos níveis de desenvolvimento e faixa etária das crianças participantes, despertando seu interesse, mostrando assim, sua utilidade na intervenção pedagógica com grupos ou até mesmo individualmente, com algumas adaptações.

- Todos os três tipos de estratégias: exploratórias, de vivência e de metalinguagem foram importantes para o trabalho em grupo com as crianças, sendo que os sujeitos da pesquisa L.V.B. e T.C. demonstraram-se mais receptivos às estratégias do tipo vivência, que envolviam o uso amplo da linguagem oral, enquanto que os sujeitos G.R.L. e S.A.B. demonstraram-se receptivos aos três tipos de estratégias, especialmente àquelas exploratórias.

- A autoestima e a confiança das crianças naquilo que produzem, neste caso, o material escrito, torna-se fundamental para os programas de reabilitação e este fato foi diariamente constatado pela pesquisadora na intervenção com as crianças.

- No grupo as crianças participaram de todas as atividades, jamais se recusando a conversar sobre as atividades propostas e dar suas opi niões sobre as leituras e produções escritas, demonstrando a importância do grupo terapêutico pedagógico para a reabilitação das crianças com deficiência auditiva, as quais nem sempre se expõem tão naturalmente em situações menos protegidas, como no caso da sala de aula.

- O conhecimento a respeito do processo de desenvolvimento individual e as importantes relações com a autoestima da criança devem alcançar as salas de aula, nas quais nem sempre os professores podem ter a visão do processo de desenvolvimento das crianças com necessidades especiais, neste caso, das crianças com deficiência auditiva. Assim faz-se imprescindível a orientação e parceria entre equipe de reabilitação e professor de cada criança.

\section{REFERÊNCIAS}

BALIEIRO, C.R.;TREN CHE,M.C.B. Conversando com pais: a escrita eo desenvolvimento da linguagem de crianças deficientes auditivas. In: BEVILACQUA, M.C.; MORET, A.L.M. D eficiência auditiva: conversando com familiares e profissionais da saúde. Pulso Editorial, 2005. p. 285-294.

BERNARDINO-JÚNIOR, J.A . etal. Rev. Bras. Ed. Esp. Marília, v.12, n.3, p.423-450, 2006.

BARRERA, S.D. Linguagem oral e alfabetização: um estudo sobre a variação lingüística e consciência metal ingüística em crianças da $1^{a \underline{a}}$ série do ensino fundamental. 2000. 225f. Tese (Doutorado em Educação) - Instituto dePsicologia, UniversidadedeSão Paulo, São Paulo.

BEVILACQUA, M.C.; FORMIGONI, G. A udiologia Educacional: uma opção para a criança deficienteauditiva. Pró-Fono,Carapicuíba,1998. 
BRAZOROTTO, J. S. Linguagem Escrita e H abilidades M etalingüísticas de Crianças Surdas e de Crianças com D ificuldades de A pren dizagem. 2002.155f. Dissertação (M estrado em Educação Especial) - UniversidadeFederal deSão Carlos, São Carlos.

BUENO, M. L. P. B. O ralidade e escrita: uma relação de complementaridade. 2003. Dissertação (Mestrado em Educação)- UniversidadeEstadual deCampinas, Campinas.

BYRNE, B. Treinamento da consciência fonêmica em crianças pré-escolares: porquefazêlo equal o seu efeito? In: MARTINS, C. C. (Org.). Con sciência fonológica eal fabetização. São Paulo: Vozes, p 39-67, 1995.

CA PELLINI, S.A ; PADULA, C.; CIA SCA, S.M. Eficácia do programa detreinamento coma Consciência Fonológica em crianças com distúrbio de aprendizagem, Pró-F ono, São Paulo, v.16, n.3, 261-274, 2004.

CA POVILLA, A. G. S.; CA POVILLA, F. C. Problemas deleitura e escrita. São Paulo: Memnon, FAPESP, 2000.

CA POVILLA , A. G. S.; CAPOVILLA, F. C. A lfabetização: método fônico. São Paulo:M emnon, 2002.

DESJARDIN, J. L.; AM BROSE, S. E.; EISEN BERG, L. S. Literacy skills in children with cochlear implants: the importance of early oral languageand joint storybook reading. Journal of $D$ eaf Studies and D eaf Education A dvance. A pril, 2008.

FRAN ÇA, M. P. et al. A quisição da linguagem oral. Relação erisco para linguagem escrita. A rq N europsiquiatr, São Paulo, 62, 2-B, 469-472, 2004.

FERREIRO, E. R eflexões sobre alfabetização. São Paulo: Cortez, 1985.

FERREIRO, E; TEBEROSKY, A. Psicogênese da língua escrita. Porto Alegre: Artes Médicas, 1986.

GILLON , G.; DOOD, R. Enhancing thephonological processing skills of children with specific reading disability. Eu ropean J ournal of Communication D isorders, s.l., v. 32 n. 2, p. 67-90, 1997.

GÓES, M.C. R.; ARA GÃO, R. M. R. Níveis decompreensão da linguagem escrita em crianças com atraso escolar. A rquivos Brasileiros de Psicologia, Rio de Janeiro, 44, 3,4, 1992.

GROSSI , E. P. A Ifabetização em classes populares: avaliação cognitiva. Porto A legre: GEEM PA, 1987.

GROSSI, E. P. D idática do nível pré-silábico. Porto A legre: publicações GEEM PA, 1988.

JARDINI, R. S. R.; SOUZA, P.T. Alfabetização ereabilitação dos distúrbios deleitura/ escrita por metodologia fono-vísuo-articulatória. Pró-Fono Revista deA tualização Científica, Barueri, v. 18, n. 1, p. 69-78, 2006.

JAMES, D.; RAJPUT, K.; BRINTON, J.GOSWAMI, U. Phonological A wareness, vocabulary, and word reading in children who use cochlear implants: does age of implantation explain individual variability in performance outcomes and growth? J ournal of D eaf Studies and D eaf Education A dvance, 2007.

LEITÃO, S.; HOGBEN, J.; FLETCHER, J. Phonological processing skills in speech and languageimpaired children. European Journal of Communication D isorders, v.32, s.n., p. 73-93, 1997. 
LOPES, L. B. O. Desenvolvimento da língua escrita no deficienteauditivo. In: ENCONTRO DE ALFABETIZADORESDE DEFICIENTESAUDITIVOS, 2., 1989, Rio de Janeiro.

MILLER, P.F. Syntactic and semantic processing in Hebrew readers with prelingual deafness. A merican A nnals of the D eaf, 145,5, 436-451, 2000.

MOORES, D. F. Psycholinguistics and deafness. A merican A nnals of the D eaf, v.142, n. 3, p.8089, 1997.

PETRECHEN, D.R.D.D esenvolvimento da escrita em crianças surdas. 2001. 203f. Dissertação (M estrado em Educação Especial) - Universidade Federal de São Carlos, São Carlos.

PINHEIRO, A.M.V.. Leitura e Escrita: uma abordagem cognitiva. Campinas: Editorial Psy., 1994.

POSKIPARTA, E.; NIEMI, P.; VAURAS, M. Who benefits from training in linguistic awareness in the first grade, and what components show training effects? Journal of Learning Disabilities, v.32, n.5, p. 437-456, 1999.

SALGADO, C.; CAPELLINI, S. A. Desempenho em leituraeescrita deescolares com transtorno fonológico. Desempenho em leitura eescrita com transtornos fonológicos. Psicologia E scolar eE ducacional, Campinas, v.8, n.2, 179-188, 2004.

SCHNEIDER, W. et al. Kindergarten prevention of dyslexia: Does training in phonological awareness work for everybody? J ournal of Learning D isabilities, 32, 5, 429-436, 1999.

TRENCHE, M.C.B. A inclusão da criança surda no ensino comum. D istúrbios da Comunicação, Campinas, p. 10-19, 1998.

VALÉRIO, T.M.M. A estimulação da consciência fonológica em in divíduos portadores de dislexia. 1998. Dissertação (M estrado em Educação) - Universi dade Estadual do Rio de Janeiro, Rio deJaneiro.

ZORZI, J. L. Consciência fonológica, fases da construção da escrita eseqüência deapropriação da ortografia do Português. In: MARCHESAN, I. Q.; ZORZI, J. L. A nuário Cefac de Fonoaudiologia. São Paulo: Revinter, 2000, p. 91-104.

ZORZI, J. L. A prender a escrever: a apropriação do sistema ortográfico. Porto Alegre: A rtes Médicas, 1998. 